
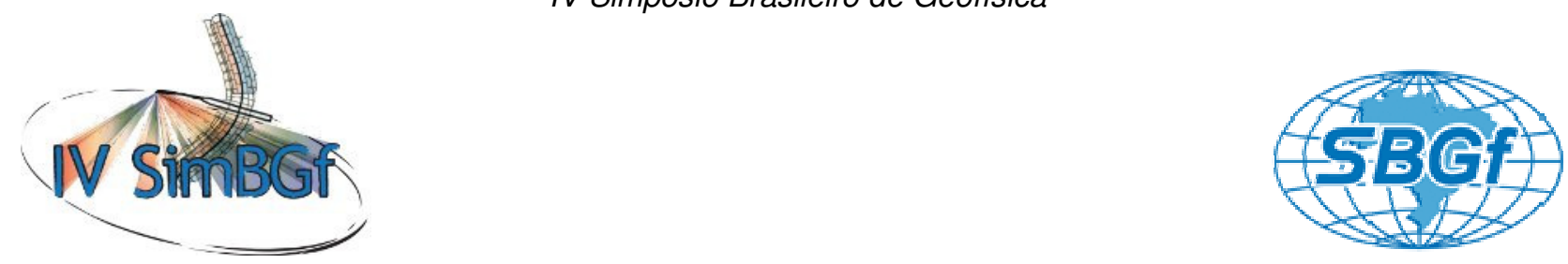

\title{
Caracterização petrofísica de rochas reservatório por Ressonância Magnética Nuclear
} Edmilson Helton Rios ${ }^{1}$, Giovanni Chaves Stael ${ }^{1}$, Paulo Frederico de Oliveira Ramos ${ }^{2}$, Vinicius de França Machado ${ }^{2}$, Ruan Barbosa Forecchi ${ }^{3}$, *Rodrigo Bagueira de Vasconcellos Azeredo ${ }^{3}$

${ }^{1}$ Observatório Nacional, ${ }^{2}$ PETROBRAS, ${ }^{3}$ Universidade Federal Fluminense

Copyright 2010, SBGf - Sociedade Brasileira de Geofísica

Este texto foi preparado para a apresentação no IV Simpósio Brasileiro de Geofísica, Brasília, 14 a 17 de novembro de 2010. Seu conteúdo foi revisado pelo Comitê Técnico do IV SimBGf, mas não necessariamente representa a opinião da SBGf ou de seus associados. É proibida a reprodução total ou parcial deste material para propósitos comerciais sem prévia autorização da SBGf.

\section{Resumo}

O presente trabalho demonstra a aplicação da técnica de RMN na caracterização petrofísica de rochas reservatório. Para isso, foram medidos os tempos de relaxação $\left(T_{1}\right.$ e $\left.T_{2}\right)$ e as constantes de difusão translacional (D) de 25 amostras de afloramentos saturadas com solução salina. A partir dos dados de RMN, propriedades como porosidade, distribuição do tamanho de poros, saturação irredutível de água, permeabilidade, entre outras, foram estimados quantitativamente e qualitativamente. Os resultados foram confrontados com valores de referências.

\section{Introdução}

Desde a descoberta do fenômeno no final da década de 40, a Ressonância Magnética Nuclear (RMN) vem se consolidando como um dos métodos indiretos mais importantes para a caracterização de propriedades petrofísicas de rochas reservatório, e também de seus fluidos saturantes (Kleinberg \& Jackson, 2001).

Parte da importância da perfilagem por RMN na avaliação de formações reside na grande quantidade de respostas que a ferramenta é capaz de fornecer, principalmente quando comparada aos perfis tradicionais. (Ellis \& Singer, 2008). Essa característica, aliada a simplicidade, rapidez de execução e o caráter não destrutivo da análise, explica parte do seu notável crescimento como ferramenta laboratorial.

Com o intuito de demonstrar sua aplicação em petrofísica, nesse artigo são apresentados resultados de diversos ensaios laboratoriais, qualitativos e quantitativos, realizados em plugues de afloramentos, que reproduzem características de rochas reservatório. Uma vez que a teoria básica de RMN encontra-se extensamente documentada na literatura (Gil \& Geraldes, 1987; Levitt, 2001), apenas uma síntese dos principais fundamentos será apresentada a seguir.

\section{Fundamentos básicos de RMN}

Quando submetidos a um campo magnético estático $B_{0}$, os momentos magnéticos nucleares de certos isótopos atômicos se alinham em movimento de precessão ao redor desse campo, dentre os quais, $0{ }^{1} \mathrm{H}$ presente na composição molecular dos fluidos de reservatório (água, óleo e gás). Esse processo, denominado polarização, produz uma magnetização resultante $M_{0}$ que aponta na direção campo $\mathrm{B}_{0}$. Essa magnetização permanece alinhada indefinidamente, até ser defletida por um pulso de radiofreqüência $B_{1}$, aplicado perpendicularmente a $B_{0}$. Ao término do pulso, $M_{0}$ retorna ao seu alinhamento original num processo denominado relaxação magnética nuclear.

Durante o processo de relaxação, uma corrente elétrica, amortecida, que constitui o sinal de RMN, é induzida numa bobina de detecção por $\mathrm{M}_{0}$. O fenômeno de relaxação envolve dois processos exponenciais distintos: o decaimento do sinal de RMN, regido pela constante de tempo $\mathrm{T}_{2}$ (tempo de relaxação transversal) e; a recuperação do alinhamento inicial de $M_{0}$, regido pela constante de tempo $T_{1}$ (tempo de relaxação longitudinal).

Os fluidos confinados no espaço poroso estão sujeitos a três mecanismos principais de relaxação (Coates et al., 2001), que são: (i) intrínseco (ou "bulk"), equivalente à relaxação intrínseca das moléculas de cada tipo de fluido; (ii) superficial, equivalente à relaxação das moléculas do fluido que estão próximas da interface rocha-fluido e; (iii) difusivo (mecanismo que afeta exclusivamente $T_{2}$ ), resultado do movimento difusivo das moléculas em presença de um gradiente de campo magnético.

Entretanto, quando o mecanismo de relaxação superficial predomina sobre os demais, as taxas de relaxação do fluido encerrado no meio poroso dependem da composição química da matriz porosa e da razão da superfície/volume, da seguinte maneira:

$$
1 / T_{1}=\rho_{1}(S / V)_{\text {poro }} \text { e } 1 / T_{2}=\rho_{2}(S / V)_{\text {poro }}
$$

na qual, $\rho_{1}$ e $\rho_{2}$ são, respectivamente, a relaxatividade superficial (propriedade inerente da matriz porosa) para $\mathrm{T}_{1}$ e $\mathrm{T}_{2}$ e; S/V é a razão superfície/volume do poro.

As Eqs.1 e 2 evidenciam o potencial da RMN de responder simultaneamente tanto à composição da matriz quanto à distribuição de tamanho dos poros, característica única quando comparada aos demais métodos indiretos (Toumelin et al., 2003).

\section{Metodologia \\ Caracterização e preparação das amostras}

Para a realização desse estudo foram selecionados 25 plugues cilíndricos $(3,8 \mathrm{~cm} \times 5,0 \mathrm{~cm}$,) bem consolidados de diversas porosidades $(3,4$ a $33,6 \%)$ e permeabilidades absolutas (30 a $9.390 \mathrm{mD}$ ), dos quais 14 oriundos de afloramentos na formação Botucatu (Brasil) e demais de 
afloramentos da formação Berea (EUA) (Gomes et al., 1996). As amostras foram limpas por extração contínua por solventes de acordo com procedimentos recomendados pelo American Petroleum Institute (APIRP40, 1998).

A porosidade total ( $\left.\phi_{\text {total }}\right)$ e permeabilidade absoluta $\left(\mathrm{K}_{\mathrm{abs}}\right)$ foram medidas a $500 \mathrm{PSI}$ e $25^{\circ} \mathrm{C}$ num porosímetro UltraPore 300 e permeabilímetro Ultraperm 500 (ambos da Core Lab, EUA). Em seguida os plugues foram completamente saturados com solução salina $(\mathrm{NaCl}$ $50 \mathrm{Kppm})$ por aplicação de vácuo, para as amostras de alta permeabilidade $\left(\mathrm{K}_{\mathrm{abs}}>400 \mathrm{mD}\right)$, ou vácuo seguido por pressurização a 2.000PSI, para amostras de baixa permeabilidade $\left(\mathrm{K}_{\mathrm{abs}}<400 \mathrm{mD}\right)$. Os plugues foram mantidos submersos na solução salina até a realização dos ensaios de RMN.

\section{Medidas de RMN}

As medidas de relaxação $\left(T_{1}\right.$ e $\left.T_{2}\right)$ e da constante de difusão translacional (D) foram realizadas a $35^{\circ} \mathrm{C}$ em dois equipamentos distintos do tipo Maran Ultra (Oxford Instruments, Reino Unido), um operando a 460Gauss, $\left(2 \mathrm{MHz}\right.$ para freqüência do $\left.{ }^{1} \mathrm{H}\right)$ e outro de operando a 3KGauss $\left(13 \mathrm{MHz}\right.$ para freqüência do $\left.{ }^{1} \mathrm{H}\right)$, ambos equipados com sondas de $52 \mathrm{~mm}$. A seqüência de pulso utilizada para a medição do $T_{2}$ foi a CPMG (Carr-PurcellMeiboom-Gill).

A Fig.1 apresenta as seqüências de pulsos híbridas empregadas na realização dos experimentos bidimensionais $T_{1}-T_{2}$ e $D-T_{2}$ (Song et al., 2002; Sun \& Dun, 2005). Elas resultam da fusão entre a seqüência CPMG, empregada na etapa de aquisição dos sinais, e as seguintes técnicas: Inversão-Recuperação, para obtenção dos mapas $\mathrm{T}_{1}-\mathrm{T}_{2}$ (Fig.1a) e; Pulsed Field Gradient Stimulated-Echo (PFG-SE), para os mapas D-T2 (Fig1b)

(a) Inversão-Recuperação

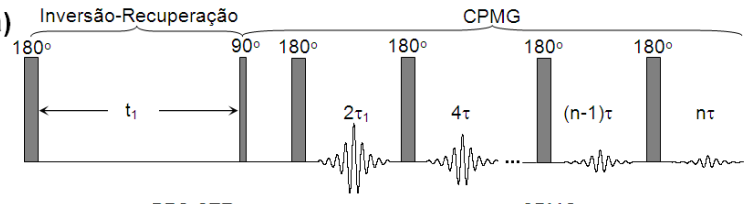

(b)

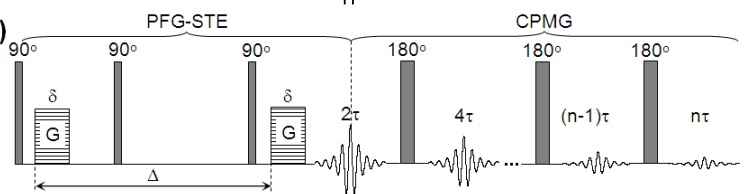

Figura 1 - Seqüências de pulso híbridas utilizadas para a obtenção dos mapas bidimensionais (a) $T_{1}-T_{2}$ e (b) $D-T_{2}$.

\section{Processamento dos dados}

As distribuições de $T_{2}$ foram obtidas através do cálculo da transformada inversa de Laplace das medidas de CPMG, utilizando-se o programa WinDXP ver.1.8.1.0 (Oxford Instruments, Reino Unido). Os mapas bidimensionais $T_{1}-T_{2}$ e $D-T_{2}$ foram obtidos através de rotinas desenvolvidas em Matlab (MathWorks, EUA) e patenteadas pela Schlumberger.

\section{Resultados e Discussão}

\section{Petrofísica básica}

A Fig.2 sumariza o resultado da caracterização petrofísica básica das amostras ensaiadas, porosidade total e permeabilidade absoluta, obtidas pelos métodos de referência.

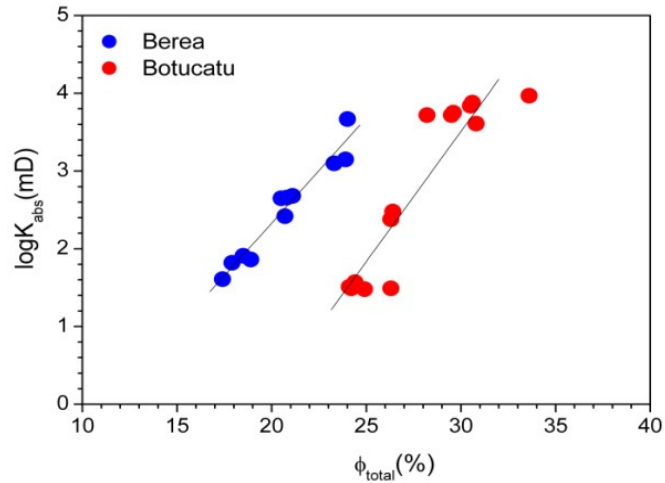

Figura 2 - Permeabilidade absoluta versus porosidade total.

Embora a permeabilidade apresente uma dependência bastante complexa de vários outros parâmetros (Schon, 2004) quando agrupadas de acordo com a litologia, a sua correlação direta com a porosidade torna-se bastante evidente.

Uma vez caracterizadas, as amostras foram analisadas por $\mathrm{RMN}$ a $2 \mathrm{MHz}$, freqüência máxima geralmente empregada na ferramentas de perfilagem, e os resultados obtidos comparados aos valores de referência.

\section{Porosidade total}

Os ${ }^{1} \mathrm{H}$ presentes na estrutura química na matriz rochosa não são detectados nas condições ajustadas $\left(T_{2}\right.$ muito curto, ou $\mathrm{T}_{1}$ muito longo), logo a amplitude inicial do sinal de $\mathrm{RMN}$, ou a área total do espectro de relaxação, é uma expressão direta apenas do volume de fluidos presentes na rocha (Zhang et al., 2000). Dessa forma, aplicando um fator de conversão apropriado, calculado a partir da medição de um volume conhecido de fluido, foi possível estimar com excelente exatidão a porosidade total por RMN, conforme mostrado na Fig.3.

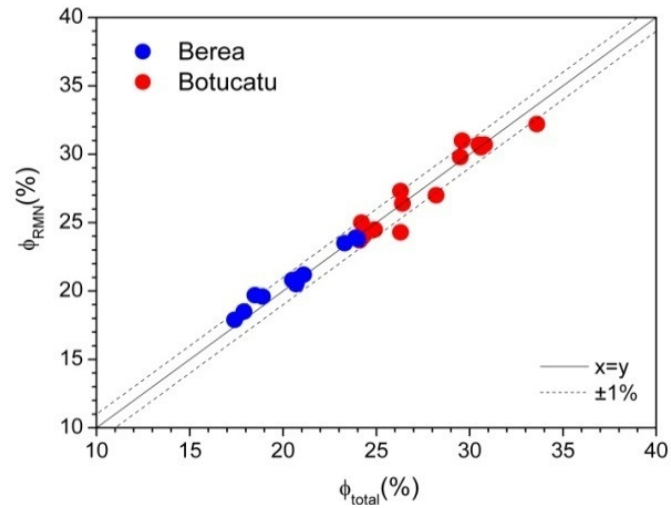

Figura 3 - Porosidade total medida pelo método de referência versus predita por RMN. 
Mesmo se tratando de litologias distintas, a porosidade por RMN foi estimada a partir de um único fator de calibração. Nesse contexto, pode-se afirmar que a RMN é um método indireto capaz de produzir estimativas de porosidade com boa exatidão e independes da litologia. Nesse caso, o desvio médio em relação aos valores de referência foi menor do que uma unidade de porosidade.

\section{Distribuição do tamanho de poros}

A Fig.4a mostra do sinal de RMN da solução salina na sua forma livre e confinada no espaço poroso. Além das diferenças na amplitude do primeiro ponto, diretamente relacionadas à porosidade, é possível observar o decaimento do sinal acelerado pela interação entre o fluido e a superfície do poro.
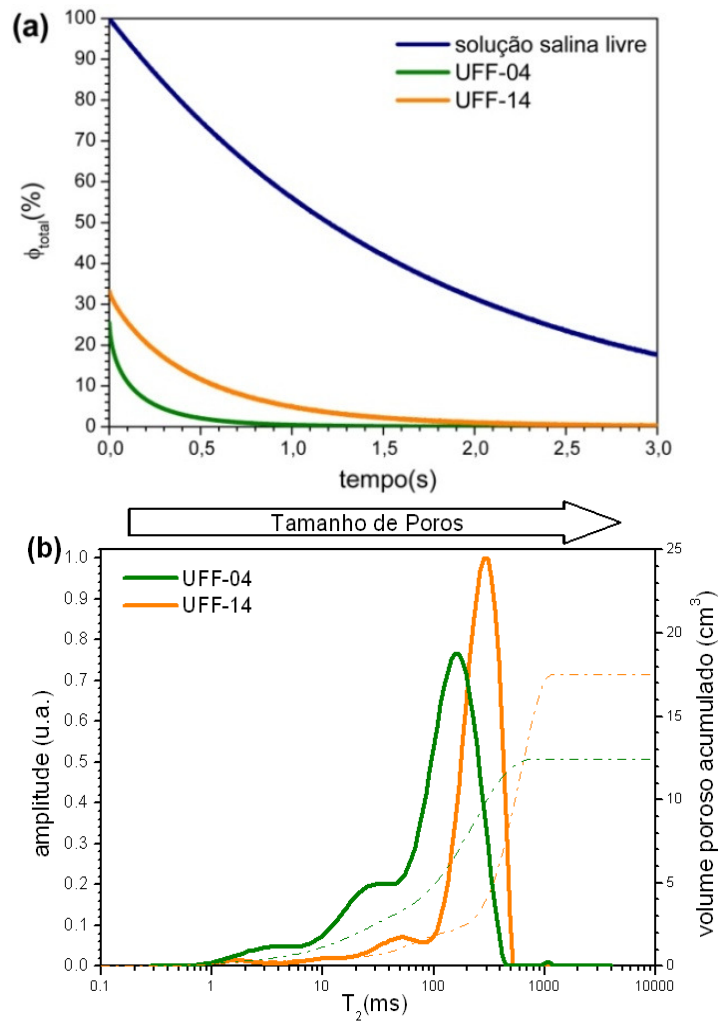

Figura 4 - (a) Decaimento do sinal de RMN da solução salina livre e contida nas amostras UFF-14 ( $\phi_{\text {total }}=33,3 \%$ e $\left.K_{a b s}=9.390 \mathrm{mD}\right)$ e UFF-04 $(25,5 \%$ e $K=30 \mathrm{mD})$ e; (b) suas respectivas distribuições de $T_{2}$.

Considerando que a relaxação é influenciada pela razão S/V dos poros (Eq.1 e 2), e que meios porosos naturais geralmente não apresentam um tamanho único de poro, espera-se que o decaimento do sinal de RMN de um fluido confinado numa rocha seja multiexponencial.

Invertendo os decaimentos do sinal RMN (Dunn et al., 2002), obtêm-se distribuições de $T_{2}$, ou espectros, que guardam uma relação direta com a distribuição de tamanho dos poros ocupados pelo fluido, Fig.4b. Neles é possível observar pelo menos três modas distintas, representando diferentes classes de tamanhos de poro. Além disso, é possível constatar que a amostra mais permeável apresenta um espectro deslocado para a direita ( $T_{2}$ mais longos), sugerindo a ocorrência de poros maiores do que os presentes na amostra de menor permeabilidade.

A conversão da escala de relaxação para a escala métrica depende do conhecimento da relaxatividade superficial da matriz. Entretanto, para meios porosos naturais, esse parâmetro é de difícil determinação experimental, e sua aplicação prática bastante limitada.

\section{Saturação irredutível de água}

A saturação irredutível de água (Swi) pode ser estimada pela separação do espectro de $\mathrm{T}_{2}$ em dois domínios limitados por um valor um valor de corte (Timur, 1968). A área do espectro abaixo do $\mathrm{T}_{2}$ de corte corresponde ao volume de fluidos aprisionados por forças capilares (bound volume irreductible - BVI), enquanto que área acima do $\mathrm{T}_{2}$ de corte corresponde ao volume de fluido livre (free-fluid index - FFI), conforme ilustrado na Fig.5.

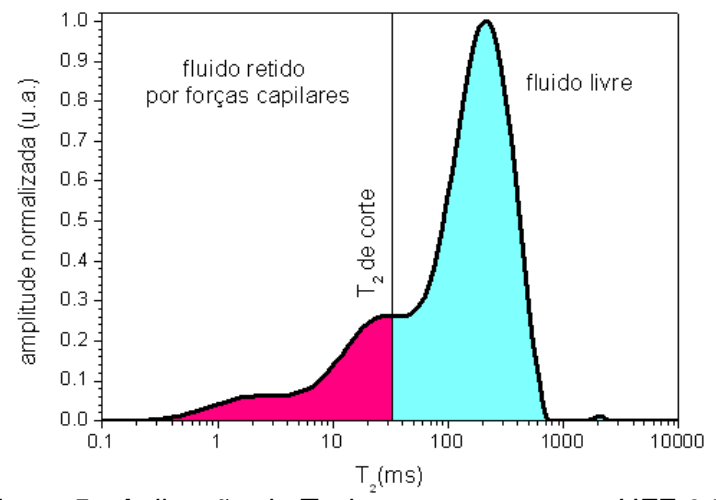

Figura 5 - Aplicação do $T_{2}$ de corte na amostra UFF-04.

Aplicando o valor de $T_{2}$ de corte sugerido na literatura para rochas siliciclástincas $(33 \mathrm{~ms})$, foi estimada a saturação de irredutível de água de cada uma das amostras. Para verificar a consistência dos resultados confrontaram-se os valores de Swi com a permeabilidade. A Fig.6 mostra os resultados obtidos.

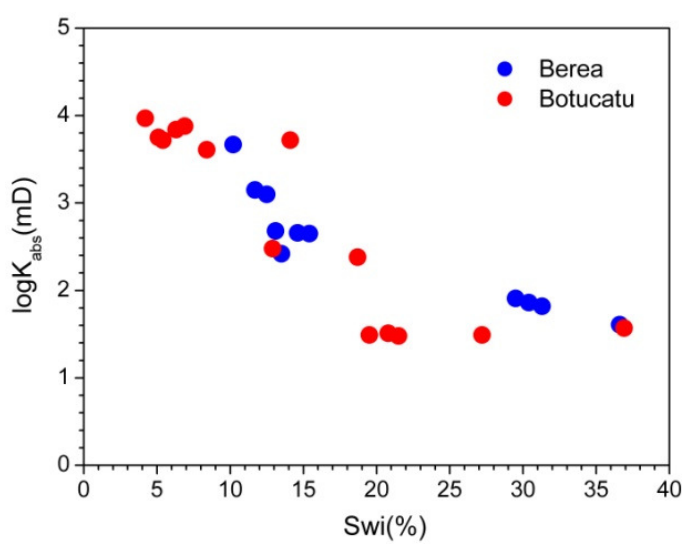

Figura 6 - Saturação irredutível de água obtida por RMN versus permeabilidade absoluta.

A tendência de aumento do Swi obtido por RMN com a diminuição da permeabilidade é coerente, uma vez que, 
quando maior a incidência de poros pequenos (área do espectro à esquerda do $\mathrm{T}_{2}$ de corte), maior será a tendência do meio poroso de resistir à passagem do fluido.

\section{Permeabilidade absoluta}

As equações Schlumberger-Doll-Research (SDR) (Kenyon et al., 1986) e de Timur-Coates (TC) (Coates et al., 1991) são consideradas as equações clássicas de estimativa de permeabilidade a partir de dados de RMN:

$$
\mathrm{K}_{\mathrm{SDR}}=\mathrm{aT}_{2 \mathrm{gm}}^{\mathrm{b}} \phi^{\mathrm{c}} \quad \text { e } \mathrm{K}_{\mathrm{TC}}=(\mathrm{FFl} / \mathrm{BVI})^{\mathrm{a}}(\phi / \mathrm{c})^{\mathrm{b}}
$$

na qual, $\mathrm{T}_{2 g m}$ é média geométrica dos tempos $\mathrm{T}_{2} \mathrm{e} ; a, b$ e $c$ são os coeficientes de ajuste litológicos.

Ambos os modelos utilizam a premissa de que o espectro de $T_{2}$ representa a distribuição de tamanho de poros e seu desempenho é reconhecido em rochas homogêneas (Kleinberg, 1996). A Fig.7 mostra o resultado das estimativas obtidas a partir das Eqs. 3 e 4, após o ajuste dos coeficientes $a, b$ e $c$.
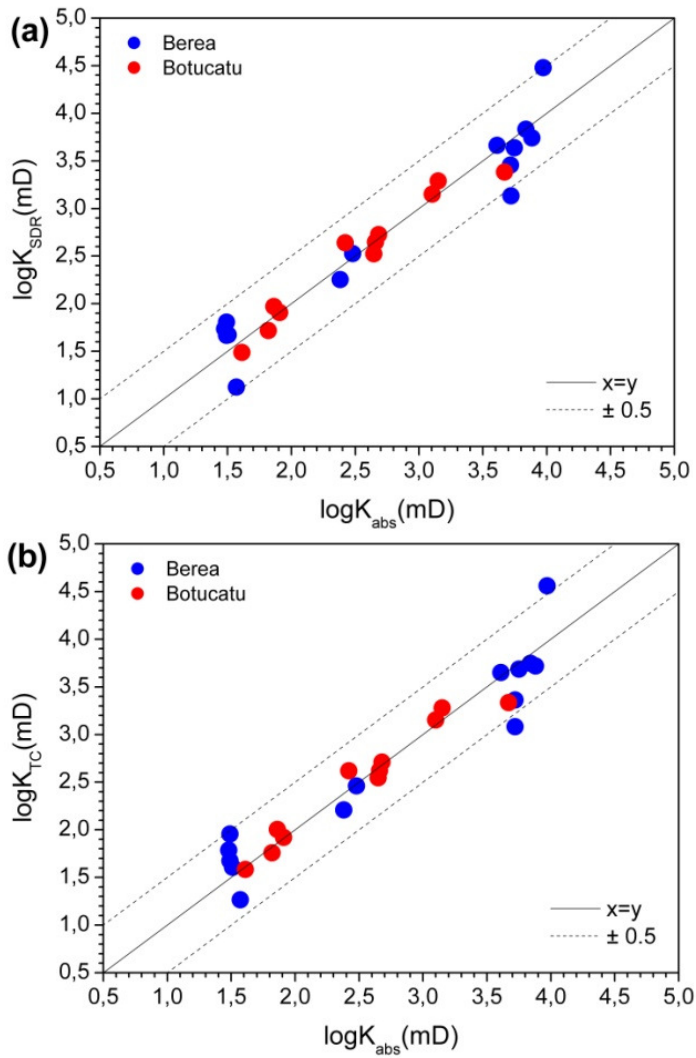

Figura 7 - Permeabilidade absoluta medida pelo método padrão versus a predita por RMN empregando os modelos SDR e TC, com os coeficientes ajustados.

Para ambos os modelos, SDR (Fig7.a) e TC (Fig.7b), o desvio médios em relação as valores de referência foi menor do que uma ordem de grandeza. Vale ressaltar que, tais modelos não têm a pretensão de estimar a permeabilidade com exatidão, pois se trata de uma propriedade complexa e com uma escala bastante dilatada (Ellis \& Singer, 2008). Por exemplo, na atividade de exploração de hidrocarbonetos, conhecer a ordem de grandeza de propriedades como a permeabilidade, ou viscosidade, muitas vezes basta para auxiliar no processo de tomada de decisões.

Mapas bidimensionais $T_{1}-T_{2}$

Para a maioria das amostras ensaiadas, os mapas $T_{1}-T_{2}$ apresentaram uma faixa estreita de variação para razão $T_{1} / T_{2}$, com valores compreendidos entre 1 e 2 , conforme exemplificado na Fig.8a.

Quando existe apenas um fluido saturando o meio poroso, a razão $T_{1} / T_{2}$ dependerá exclusivamente de eventuais diferenças na composição química da superfície do poro (Allen et al., 1996). Por essa razão, os resultados obtidos sugerem que, ao menos em sua superfície, as amostras apresentam uma composição química homogênea.
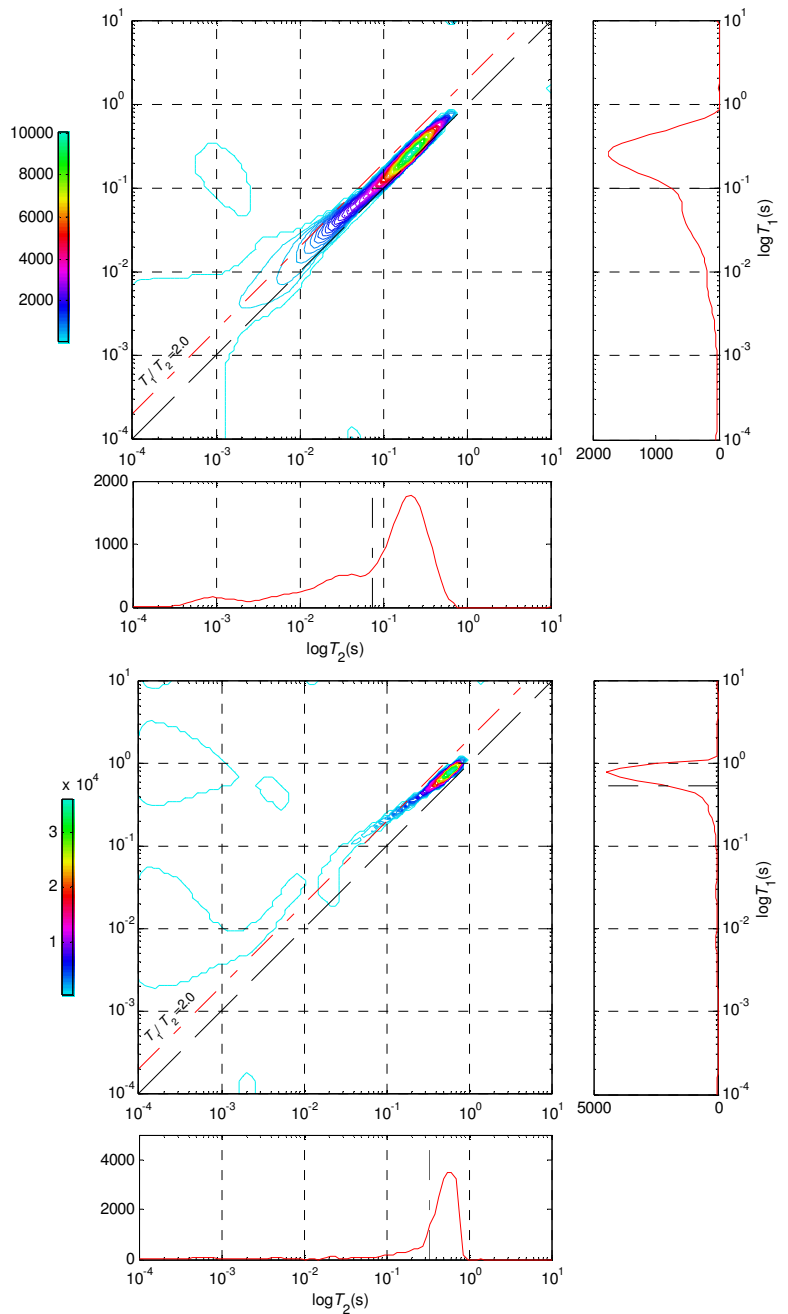

Figura 8 - Mapa $T_{1}-T_{2}$ para as mostras (a) UFF-04 e; (b) UFF-16.

Entretanto, para um pequeno grupo de amostras os mapas $T_{1}-T_{2}$ apresentaram uma correlação distorcida e com uma faixa de variação para razão $T_{1} / T_{2}$ bem mais 
ampla, com valores entre 4 e 1. A Fig.8b exemplifica esse comportamento para a amostra UFF-16.

Um levantamento posterior revelou que as amostras que apresentaram esse comportamento são oriundas de um ensaio de molhabilidade, onde foram impregnadas com um agente químico que, mesmo após a limpeza subseqüente, deixa resíduo.

Dessa forma, supõe-se que, apesar da litologia da matriz ser a mesma, a superfície do poro está parcialmente recoberta por um agente químico que, apesar de não alterar suas características petrofíscas básicas, interage com o fluido saturante modificando o seu tempo de relaxação.

\section{Mapas bidimensionais $D-T_{2}$}

Os mapas $\mathrm{D}-\mathrm{T}_{2}$ apresentaram duas correlações bastante distintas, representados na Fig.9 pelas amostras UFF-04. A primeira, bem mais intensa, correlaciona um $T_{2}$ mais longo com uma constante de difusão translacional $D$ característica para água livre. Essa correlação sugere a predominância de poros grandes, quando comparados aos demais, onde o fluido se difunde livremente.

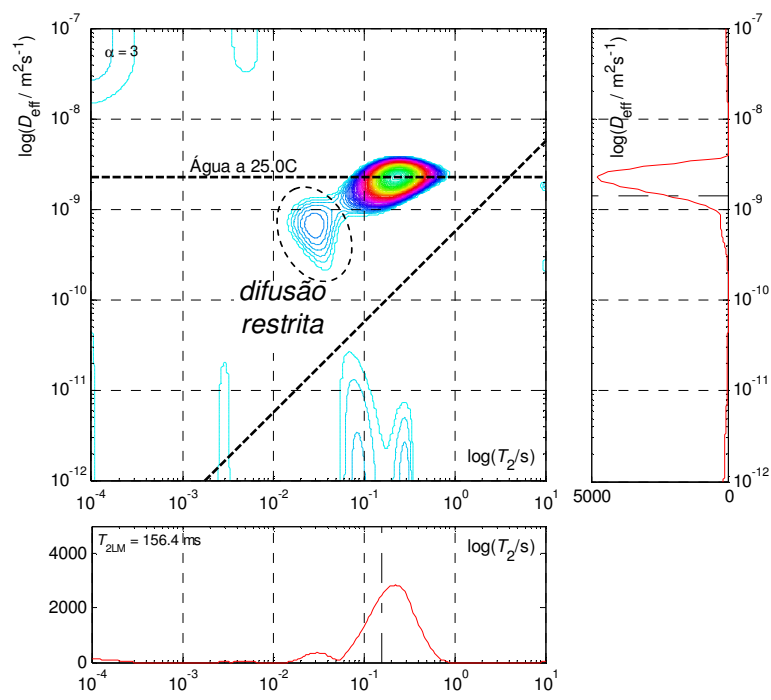

Figura 9 - Mapa $D-T_{2}$ para a mostra UFF-04. Em destaque a região associada à difusão restrita.

A segunda correlação, menos intensa, corresponde a tempos $T_{2}$ mais curtos e constantes de difusão $D$ bem menores do que o valor da água livre. Tal fato sugere a ocorrência de difusão restrita, típico para poros menores, onde o movimento de difusão translacional é impedido pela parede do poro (Bergman et. al., 1995; Valfouskaya et. al. 2005).

Esses mapas também são particularmente úteis quando dois fluidos coexistindo no mesmo espaço poroso apresentam tempos de relaxação sobrepostos, por exemplo, água e óleo leve. Nesses casos é possível discriminá-los com base nas diferenças de D.

\section{Avaliação de gradientes de campo magnético}

Com o propósito de avaliar qualitativamente o efeito de gradientes de campo magnético no interior do poro, medidas adicionais de $T_{2}$ foram realizadas a $13 \mathrm{MHz}, 0$ que equivale a um campo magnético 6,5 vezes mais intenso do que o anterior.

Os resultados demonstraram que, para as amostras de Botucatu, os espectros obtidos a 2 e $13 \mathrm{Mz}$ não apresentaram diferenças significativas, conforme exemplificado na Fig.10a para a amostra UFF-21. Para as amostras de Berea, embora a forma dos espectros a $13 \mathrm{MHz}$ não tenha se alterado, foi observado um significativo deslocamento para tempos de relaxação mais curtos, conforme mostrado na Fig.10b para a amostra UFF-31.
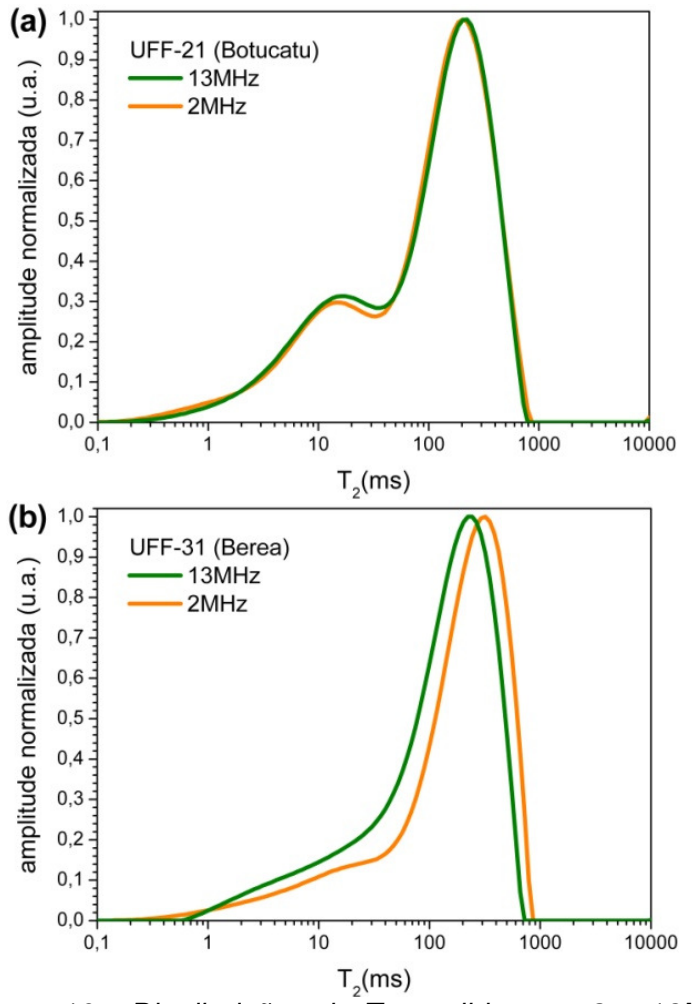

Figura 10 - Distribuições de $T_{2}$ medidas em 2 e $13 \mathrm{MHz}$ para as amostras (a) UFF-21 do tipo Botucatu e; (b) UFF31 do tipo Berea.

Especula-se que, nas amostras de Berea, gradientes de campo no interior do poro, gerados pela diferença entre a suceptibilidade magnética da matriz sólida e do fluido (Dunn et al., 2002), podem estar produzindo uma via de relaxação adicional à relaxação superficial. Uma vez que esses gradientes são diretamente proporcionais à intensidade do campo externo aplicado, a $2 \mathrm{MHz}$ esse efeito é inibido.

Embora a composição mineralógica dessas amostras ainda não esteja disponível, esses resultados preliminares sugerem uma menor ocorrência de minerais paramagnéticos nas amostras de Botucatu, quando comparado as de Berea. Por essa razão, mesmo quando submetidos a campos magnéticos mais intensos, os gradientes internos não sejam tão significativos nas amostras de Botucatu. 


\section{Conclusões}

Embora esse trabalho não tenha a pretensão de esgotar o tema, foi demonstrada aplicabilidade da técnica de RMN na caracterização, quantitativa e qualitativa, de importantes propriedades petrofísicas de amostras de rocha saturadas com um único fluido.

Utilizando um fator de calibração único, independente da litologia investigada, foi possível de estimar com excelente exatidão a porosidade total das amostras de rocha. A permeabilidade, por sua vez, devido à escala extremamente dilatada, além de sua dependência complexa de outros parâmetros (cimentação, tortuosidade, etc.), foi estimada apenas sua ordem de grandeza.

As demais caracterizações apresentaram resultados bastante coerentes, evidenciando o potencial de aplicação da técnica de RMN, seja complementando, ou até mesmo, substituindo ensaios tradicionais, mais custosos, laboriosos e destrutivos. Como por exemplo, no caso da porosimetria por injeção de mercúrio, cujo uso vem sendo limitado, e até mesmo banido, em diversos laboratórios de petrofisica, devido a questões relacionadas ao impacto ambiental e a saúde dos operadores, provocadas pelo mercúrio.

\section{Agradecimentos}

Cnpq Finep, PETROBRAS e pelo apoio financeiro. Schlumberger pela utilização das rotinas de inversão. Departamento de Geologia da UFRJ pela utilização do equipamento de RMN. Ao MSc. Andre Alves de Souza (Schlumberger) pelas valiosas discussões.

\section{Referências}

ALLEN SG, STEPHENSON PCL \& STRANGE JH. 1997. Morphology of porous media studied by nuclear magnetic resonance, J. Chem. Phys., 106(18): 7802-7809.

AMERICAN PETROLEUM INSTITUTE. 1998.
Recommended practices for core
Recommended Practice RP40, 2nd ed., 220 pp.

BERGMANN DJ, DUNN KJ, SCHWARTZ LM \& MITRA PP. 1995. Self-diffusion in periodic porous medium: a comparison of different approaches, Phys. Rev. E, 51(4): 3393-3400.

COATES GR, MILER M, GILLEN M \& HENDERSON G. 1991. The MRIL in Conoco 33-1: an investigation of a new Magnetic Resonance Imaging log. Annu. Log. Sym. SPWLA, 32: 1-24.

COATES G, XIAO L \& PRAMMER M. 2001. NMR logging principles and applications. Gulf Professional Publishing, Houston, 234pp.

DUNN KJ, BERGMAN, DJ \& LATORRACA GA. 2002. Nuclear Magnetic Resonance Petrophysical and Logging Applications. Elsevier, Amsterdam, 293pp.
ELLIS DW \& SINGER JM. 2008. Well logging for earth scientists. 2nd ed., Springer, Dordrecht, pp 415-478.

Gil VMS \& GERALDES CFGC. 1987. Ressonância Magnética Nuclear: fundamentos, métodos e aplicações. Fundação Calouste Gulbenkian, Lisboa, pp 3-710.

GOMES JAT, TIBANA P \& CORRÊA ACF. 1996. Análises petrográfica e petrofisíca dos arenitos Berea e Botucatu Conexpo Arpel'96, Rio de Janeiro.

KENYON WE, DAY PI, STRALEY C \& WILLEMSEN JF. 1986. A three-part study of NMR longitudinal relaxation studies of water saturated sandstones, SPE Form. Eval., 3: 662-636.

KLEINBERG RL. 1996. Well logging, Encyclopedia of Nuclear Magnetic Resonance. John Wyley and Sons, New York, pp 4960-4969.

KLEINBERG RL \& JACKSON JA. 2001. An introduction to the history of NMR well logging. Concept. Magnetic. Res., 13(6): 340-342.

LEVITT MH. 2001. Basic principles of NMR spectroscopy. Wiley, Chichester, 686pp.

SCHON JH. 2004. Physical properties of rocks: fundamentals and principal of petrophysics. Elsevier, Amsterdam, 583pp.

SONG YQ, HURLIMANN MD, FLAUM M, FRULLA P \& STRALEY C. 2002. T1-T2 correlation spectra obtained using a fast two-dimensional Laplace Inversion. J. Magn. Reson., 154: 261-268.

SUN BQ \& DUNN KJ. 2005. Two-dimensional nuclear magnetic resonance petrophysics. Magn. Reson. Imaging, 23: 259-262.

TIMUR A. 1968. An investigation of permeability and porosity, and residual water saturation relationship for sandstone reservoirs. The Log Analyst., 9(4): 8.

TOUMELIN E \& TORRES C. 2003. Modeling of multiple echo-time NMR measurements for complex pore geometries and multiphase saturations. Soc. Petrol. Eng. J., 6: 234-243.

ZHANG Y, XIA P \& YU Y. 2000. Review of Nuclear Magnetic Resonance Magnet for Oil Well Logging. IEEE Trans. Appl. Supercond., 10:763-766. 